infection, illness, and absence from work in young, healthy healthcare professionals. The study was a randomized, prospective trial over a 3-year period (1992/19931994/1995) in two large teaching hospitals in Baltimore.

Two hundred sixty-four hospital-based healthcare professionals without chronic medical problems were recruited. A total of 359 person-winters of serological surveillance (99.4\% follow-up) and 4,746 person-weeks of illness surveillance (100\% follow-up) was conducted. Twenty-four (13.4\%) of 179 control subjects and $3(1.7 \%)$ of 180 influenza vaccine recipients had serological evidence of influenza type A or B infection during the study period. Vaccine efficacy against serologically defined infection was $88 \%$ for influenza $A$ and $89 \%$ for influenza B. Among influenza vaccinees, cumulative days of reported febrile respiratory illness were 28.7 per 100 subjects compared with 40.6 per 100 subjects in controls, and days of absence were 9.9 per 100 subjects versus 21.1 per 100 subjects in controls.

The authors concluded that influenza vaccine is effective in preventing infection by influenza $A$ and $B$ in healthcare professionals and may reduce reported days of work absence and febrile respiratory illness. These data support a policy of annual influenza vaccination of healthcare professionals.

FROM: Wilde JA, McMillan JA, Serwint J, Butta J, O'Riordan M, Steinhoff MC. Effectiveness of influenza vaccine in health care professionals: a randomized trial. JAMA 1999;281:908-913.

\section{HIV Rarely Transmitted by Saliva}

Oral transmission of HIV by the millions of HIV-infected individuals is a rare event, even when infected blood and exudate is present. Saliva of viremic individuals usually contains only noninfectious components of HIV, indicating virus breakdown.

Because most of the infectious HIV that is shed mucosally by asymptomatic individuals is found in, produced by, and transmitted by infected mononuclear leukocytes, Baron and colleagues conducted a study to determined whether saliva, which is hypotonic, may disrupt these infected cells, thereby preventing virus multiplication and cell-to-cell transmission of HIV. Specifically, they measured whether mononuclear leukocytes were lysed by saliva and whether the lysis by saliva inhibits the mul- tiplication of HIV and other viruses in infected leukocytes and other cells.

The results indicated that saliva rapidly disrupted $90 \%$ or more of blood mononuclear leukocytes and other cultured cells. Concomitantly, there was a 10,000-fold or higher inhibition of the multiplication of HIV and surrogate viruses. Further experiments indicated that the cell disruption is due to the hypotonicity of saliva.

Thus, hypotonic disruption may be a major mechanism by which saliva kills infected mononuclear leukocytes and prevents their attachment to mucosal epithelial cells and production of infectious HIV, thereby preventing transmission.

FROM: Baron S, Poast J, Cloyd MW. Why is HIV rarely transmitted by oral secretions? Arch Intern Med 1999;159:303-310.

\section{MRSA at a National Cystic Fibrosis Center}

In many patient populations, there has been a progressive increase in the prevalence of methicillin-resistant Staphylococcus aureus (MRSA). Thomas and coinvestigators from the Imperial College School of Medicine, London, examined the prevalence and consequences of acquiring MRSA in the adult cystic fibrosis (CF) population at Royal Brompton Hospital. Patients who became colonized by MRSA between 1965 and 1997 were identified from an existing database, and case notes were reviewed. Clinical and microbiological data were recorded. Twenty-six patients became colonized with MRSA during this period. Median age at acquisition was 23.4 (range, 11.8-43.3) years. In 17 patients, MRSA isolates were first identified while under the care of a total of 11 other institutions. Since the first case of MRSA infection in 1982, there has been an increase in prevalence to a current rate of nine cases in the first 7 months of 1997. The most common site of colonization was the lower airway $(96 \%)$; the nose (23\%) and skin sites (15\%) were affected more rarely. Duration of colonization was frequently brief, with nine cases (35\%) lasting less than 1 month.

The identification of MRSA appeared to be of little clinical significance and did not generally affect outcomes. Only three patients were MRSA-positive at the time of death, and in only one of these was MRSA considered a possible contributing factor.

FROM: Thomas SR, Gyi KM, Gaya H, Hodson ME. Methicillin-resistant Staphylococcus aureus: impact at a national cystic fibrosis centre. J Hosp Infect 1998;40:203-209. 